\title{
Detection of Inter-Lineage Natural Recombination in Avian Paramyxovirus Serotype 1 Using Simplified Deep Sequencing Platform
}

\author{
Dilan A. Satharasinghe ${ }^{1,2}$, Kavitha Murulitharan ${ }^{1}$, Sheau W. Tan ${ }^{1}$, Swee K. Yeap ${ }^{1}$, \\ Muhammad Munir ${ }^{3}$, Aini Ideris ${ }^{1,4}$ and Abdul R. Omar ${ }^{1,4 *}$ \\ 1 Laboratory of Vaccine and Immunotherapeutic, Institute of Bioscience, Universiti Putra Malaysia, Serdang, Malaysia, \\ ${ }^{2}$ Faculty of Veterinary Medicine and Animal Science, University of Peradeniya, Peradeniya, Sri Lanka, ${ }^{3}$ Infection and Innate \\ Immunity Research Group, Avian Viral Diseases, The Pirbright Institute, Surrey, UK, ${ }^{4}$ Faculty of Veterinary Medicine, Universiti \\ Putra Malaysia, Serdang, Malaysia
}

\section{OPEN ACCESS}

Edited by:

Akio Adachi,

Tokushima University, Japan

Reviewed by:

Oscar Negrete

Sandia National Labs, USA

Takemasa Sakaguchi,

Hiroshima University, Japan

Kaoru Takeuchi,

University of Tsukuba, Japan

*Correspondence: Abdul R. Omar aro@upm.edu.my

Specialty section:

This article was submitted to

Virology,

a section of the journa

Frontiers in Microbiology

Received: 19 September 2016 Accepted: 15 November 2016 Published: 30 November 2016

Citation:

Satharasinghe DA, Murulitharan $K$, Tan SW, Yeap SK, Munir M, Ideris A and Omar AR (2016) Detection of Inter-Lineage Natural Recombination in Avian Paramyxovirus Serotype 1 Using Simplified Deep Sequencing

Platform. Front. Microbiol. 7:1907. doi: 10.3389/fmicb.2016.01907
Newcastle disease virus (NDV) is a prototype member of avian paramyxovirus serotype 1 (APMV-1), which causes severe and contagious disease in the commercial poultry and wild birds. Despite extensive vaccination programs and other control measures, the disease remains endemic around the globe especially in Asia, Africa, and the Middle East. Being a single serotype, genotype II based vaccines remained most acceptable means of immunization. However, the evidence is emerging on failures of vaccines mainly due to evolving nature of the virus and higher genetic gaps between vaccine and field strains of APMV-1. Most of the epidemiological and genetic characterizations of APMVs are based on conventional methods, which are prone to mask the diverse population of viruses in complex samples. In this study, we report the application of a simple, robust, and less resource-demanding methodology for the whole genome sequencing of NDV, using next-generation sequencing (NGS) on the Illumina MiSeq platform. Using this platform, we sequenced full genomes of five virulent Malaysian NDV strains collected during 2004-2013. All isolates clustered within highly prevalent lineage 5 (specifically in lineage $5 a)$; however, a significantly greater genetic divergence was observed in isolates collected from 2004 to 2011. Interestingly, genetic characterization of one isolate collected in 2013 (IBS025/13) shown natural recombination between lineage 2 and lineage 5. In the event of recombination, the isolate (IBSO25/13) carried nucleocapsid protein consist of 55-1801 nucleotides (nts) and near-complete phosphoprotein (1804-3254 nts) genes of lineage 2 whereas surface glycoproteins (fusion, hemagglutinin-neuraminidase) and large polymerase of lineage 5. Additionally, the recombinant virus has a genome size of $15,186 \mathrm{nts}$ which is characteristics for the old genotypes I-IV isolated from 1930 to 1960. Taken together, we report the occurrence of a natural recombination in circulating strains of NDV in commercial poultry using NGS methodology. These findings will not only highlight the potential of RNA viruses to evolve but also to consider the application of NGS in revealing the genetic diversity of these viruses in clinical materials. Factors that drive these evolutionary events and subsequent impact of these divergences on clinical outcome of the disease warrant future investigations.

Keywords: Newcastle disease virus, avian paramyxovirus 1, next-generation sequencing, phylogenetic analysis, recombination 


\section{INTRODUCTION}

Newcastle disease virus (NDV) is a type species of an avian paramyxovirus serotype 1 (APMV-(1), which belongs to genus Avulavirus in the family Paramyxoviridae. The virus carry negative-sense, single-stranded, and non-segmented RNA genome, which encodes for at least six structural proteins, including nucleocapsid protein (NP), phosphoprotein (P), matrix protein $(\mathrm{M})$, fusion protein $(\mathrm{F})$, haemagglutinin-neuraminidase $(\mathrm{HN})$, and large protein (L) (Chambers et al., 1986). Through the RNA editing of the $\mathrm{P}$ gene, one accessory non-structural protein (known as $\mathrm{V}$ ) is synthesized, and probability a second protein named $\mathrm{W}$ is also produced (Locke et al., 2000). The $\mathrm{HN}$ and $\mathrm{F}$ proteins are surface glycoproteins that determine the virus neutralization and protection. The NP, $\mathrm{P}$, and L proteins encapsidate the viral nucleic acid; the $M$ protein lined the virus envelope and V protein interfere with interferon (IFN) responses (Dortmans et al., 2011; Zenglei and Liu, 2015).

Depending on the pathogenicity in chicken, NDV strains are classified into highly virulent (velogenic), intermediate (mesogenic), and avirulent (lentogenic) strains (Alexander, 1997). The amino acid sequence of the F protein cleavage site has been used to determine the pathogenicity of NDV (Nagai et al., 1976). Generally, the sequence of the F protein cleavage site in mesogenic and velogenic strains of NDV consists of a polybasic cleavage site $(R / K) R Q(R / K) R \downarrow F$, which is readily recognized by ubiquitous furin, an intracellular protease abundant in several cells and tissues, and consequently cleaves in many tissues resulting in systematic infection. In contrast, initiation of viral infection by cleavage of the $\mathrm{F}$ protein in lentogenic strains is restricted to respiratory and enteric systems and thus limiting the infections in these organs (Collins et al., 1993).

Although all NDV's strains are grouped within APMV-1, yet viruses show significant genetic diversity (Alexander, 1997; Aldous et al., 2003; Kim et al., 2007a). Without any incongruity, there are two methods which are currently being used to classify APMV-1 strains into different clusters. Aldous et al. (2003) have divided APMV-1 into six lineages and 13 sub-lineages, which later included three sub-lineages (Aldous et al., 2003; Snoeck et al., 2009). According to the second system, APMV-1 has been divided into two main categories which are represented as class I and class II. The class I is also subdivided into nine genotypes while the class II is subdivided into at least 13 genotypes (BallagiPordány et al., 1996; Czeglédi et al., 2006; Kim et al., 2007b). In general, there is just slight dissimilarity that exists amid the two systems (Miller et al., 2010) in which lineage 6 comprised class I viruses and lineage 1,2, 4, and 5 consisted of viruses belonging to genotype I, II, VI, and VII of class II, respectively. Furthermore, highly diverse lineage 3 comprised of genotype III, IV, V, and VIII of class II viruses (Miller et al., 2010). Several previous studies have shown that genotype V, VI, and VII of class II viruses were the most prevalent viruses presently circulating globally. Out of these, lineage 5 or genotype VII of class II viruses were the most predominantly isolated strains of NDV from Asia, Africa, Middle East, and South America (Khan et al., 2010; Miller et al., 2010; Munir et al., 2012; Perozo et al., 2012). A recent study has classified NDV strains into several new genotypes based on the mean inter-populationary evolutionary distance of $10 \%$ as the cutoff value (Diel et al., 2012). Based on that study, viruses in class I formed a single genotype, while class II viruses were separated into 15 genotypes including 10 earlier established genotypes (I - IX and XI) and five new genotypes (X, XII, XIII, XIV, and $\mathrm{XV}$ ). The subsequent study introduced two additional genotypes into this classification system (XVII and XVIII) from NDV outbreaks occurring in the Asia, west, and central Africa (Munir et al., 2012; Snoeck et al., 2013). Taken together, none of the classification systems represent a clear lineage/genotype-specific disease potential, geographical distribution or host specifications.

Similar to other RNA's viruses, NDV holds potential for evolution (Miller et al., 2010). However, limited information is available that clearly defines events of evolution, recombination, and possible gained pathogenicities of NDV strains in avian hosts. This lack of information is partly due to (i) mainly NDVs are characterized based on the partial sequence of $F$ or $\mathrm{HN}$ genes which are insufficient to identify recombination (Song et al., 2011), (ii) full genetic characterized of majority of APMVs is performed using conventional methodologies (PCRs and Sanger sequencing) which is not only error prone but also less capable to identify poorly presented virus population in the complex samples, and (iii) initial clinical material appeared to be insufficient for amplification of full genome in conventional PCRs. This requires propagation of viruses in chicken eggs, and this process may favor the replication of fitter viruses and masking the recombined strains that by any means are lower in population. Despite these shortcomings, recombination events, and evolution diversities have been reported in the NDV (Qin et al., 2008; Chong et al., 2010). Previously, Han et al. (2008) have reported a natural recombination NDV isolate named cockatoo/Indonesia/14698/90 (AY562985) with anonymous major parental lineage and two minor parentallike lineages derived from vaccine lineage and anhinga/U.S. (Fl)/44083/93 lineage, in that order (Han et al., 2008).

One of the driving forces for recombination and increasing pathogenic potential of viruses is the immune pressure imposed by the extensive vaccination (Read et al., 2015). For NDV, the primary means of disease control has been the employment of exhaustive vaccination program in both layer and broiler poultry sectors. Despite mass vaccination being practiced, NDV outbreaks have been reported throughout the world and have led to substantial economic losses to the industry over the years (Miller et al., 2010). This escaped protection has been linked to the partial protection by the current vaccines and persistent virus shedding in the immunized birds (Xiao et al., 2012; Samuel et al., 2013).

In Malaysia, an intensive vaccination program has been placed, however, the disease is continuously emerging, and the impact is enormous (Yusoff and Tan, 2001; WAHID, 2015). In this regards, we have recently characterized several isolates of NDV from various states of Malaysia from 2004 to 2005 (Tan et al., 2010). This characterization was based on the partial F gene sequences which limit the full genetic characterization and detection of recombination events. Here, we optimized a simple, robust and less resource-demanding methodology for the whole genome sequencing of APMVs using next-generation 
sequencing (NGS) technology. Using this protocol, we generated unbiased consensus-level full genomes of five APMVs strains which were obtained from clinical outbreaks during 2004-2013 in Malaysia. Phylodynamics and evolutionary analysis revealed recombination between lineage 2 and 5 and shown features that are unique for this lineage/genotype of APMV-1. The presented results are crucial to establishing bases for the vaccine-induced immune pressures, vaccine failure, and potential of APMVs to evolve for higher pathogenicity.

\section{MATERIALS AND METHODS}

\section{Samples Collection}

Five NDV isolates were obtained from individual outbreaks in the commercial poultry farms that were vaccinated for Newcastle disease (ND) located in different states in Malaysia (Table 1). Two isolates, IBS002/11 and IBS005/11, were isolated in 2011 while other three isolates, MB128/04, MB 076/05, and IBS025/13, were isolated in 2004, 2005, and 2013, respectively. Apart from IBS025/13, all samples were detected positive previously using reverse transcriptase PCR (RT-PCR) which is based on the partial $\mathrm{F}$ gene (data not shown). In addition, these isolates have been characterized in the past as genotype VII NDV isolates which are based on the partial F gene sequencing (Berhanu et al., 2010; Tan et al., 2010; Roohani et al., 2015).

\section{Virus Specimens}

All NDVs used in this study were triple plaque-purified on chicken embryo fibroblast (DF1 cells) and propagated by inoculating into 9 days-old specific-pathogen-free (SPF) embryonated chicken eggs and stored in liquid nitrogen. Virus stocks of the selected NDV isolates were thawed and propagated in the allantoic cavity of 9 days old SPF embryonated chicken eggs according to European Community Directive 92/66/EC (CEC, 1992) and identified using hemagglutination (HA) test. Allantoic fluids from the sample showing high HA titers more than $2^{7}$ were divided into working stocks and stored at $-20^{\circ} \mathrm{C}$. These allantoic fluids were used as working stocks for pathogenicity assessments and sequencing.

\section{RNA Extraction and Quality Control}

Genomic viral RNA was extracted from the infected allantoic fluid of SPF eggs by using TRIzol ${ }^{\circledR}$ Reagent (Invitrogen, USA) according to the manufacturer's instructions. After air drying, the RNA pellet was resuspended in $40 \mu \mathrm{L}$ nucleasefree water (Ambion, USA). The quantity and purity of the extracted RNA were checked by Eppendorf BioSpectrometer ${ }^{\circledR}$ subsequently, and was then stored at $-80^{\circ} \mathrm{C}$ for further analysis.

\section{Designing of Primers}

Based on the complete genome of NDV strain chicken/Banjarmasin/010/10 (HQ697254.1), five pairs of primer sets (Table 2) were designed to amplify the whole genome of five Malaysian NDV isolates. The full genome of NDV was divided into 5 fragments encompassing hypervariable regions flanked by more conserved sequence regions. The fragments that were identified with the most conserved regions were subjected to NCBI Primer 3 and Primer-BLAST tool and five diverse primer pairs were designed. An additional primer pair was designed for the third fragment, which consists of the highly variable regions.

\section{cDNA Synthesis}

First-strand cDNA synthesis (reverse transcription) was performed using MMLV Reverse Transcriptase First-Strand cDNA synthesis kit (Epicentre, USA). Briefly, the following components were combined on ice: $6.5 \mu \mathrm{L}$ of RNase-Free Water, $5 \mu \mathrm{L}$ of viral RNA sample and $1.5 \mu \mathrm{L}$ of fragment 1 forward primer (Table 2) for a $12.5 \mu \mathrm{L}$ total reaction volume, incubated at $65^{\circ} \mathrm{C}$ for $2 \mathrm{~min}$ with heated lid and chilled on ice for $1 \mathrm{~min}$. A second reagent mix of $2 \mu \mathrm{L}$ MMLV RT $10 \times$ Reaction Buffer, $2 \mu \mathrm{L} 100$ mM DTT, $2 \mu \mathrm{L}$ dNTP PreMix, 0.5 $\mu \mathrm{L}$ RiboGuard RNase Inhibitor and $1 \mu \mathrm{L}$ MMLV Reverse Transcriptase were added to each first-strand cDNA synthesis reaction and mixed gently on the ice. The reaction was incubated at $37^{\circ} \mathrm{C}$ for $60 \mathrm{~min}$ followed by heating at $85^{\circ} \mathrm{C}$ for $5 \mathrm{~min}$ and chilled on ice for at least $1 \mathrm{~min}$. The cDNA was briefly centrifuged and immediately used or stored at $-80^{\circ} \mathrm{C}$ for future analysis.

TABLE 1 | Clinical description and vaccination history of Malaysian NDV isolates used in this study.

\begin{tabular}{|c|c|c|c|c|c|c|}
\hline NDV isolate & Year & Origin & Farm type & Case history & Vaccination history & References \\
\hline MB128/04 & 2004 & Selangor & Broiler & Not available & Not available & Berhanu et al., 2010 \\
\hline MB076/05 & 2005 & Sabah & Broiler & $\begin{array}{l}\text { Depression, marked conjunctivitis, anorexia, and facial } \\
\text { edema }\end{array}$ & Not available & Berhanu et al., 2010 \\
\hline IBS002/11 & 2011 & Johor & Broiler & $\begin{array}{l}\text { The infection started at day } 14 \text { with } 5 \% \text { mortality. Bird } \\
\text { showed signs of coughing, sneezing, and torticollis }\end{array}$ & At day 8 and 14 with live ND+IB & Roohani et al., 2015 \\
\hline IBS005/11 & 2011 & Penang & Broiler & $\begin{array}{l}\text { The infection started at day } 29 \text { with } 5 \% \text { mortality. } \\
\text { Depression and greenish diarrhea, respiratory rales }\end{array}$ & At day 2,8 , and 12 with ND+IB & Roohani et al., 2015 \\
\hline IBS025/13 & 2013 & Penang & Broiler & $\begin{array}{l}\text { The infection started at day } 18 \text { with } 4 \% \text { mortality. Bird } \\
\text { showed signs of coughing, sneezing, greenish diarrhea } \\
\text { and torticollis }\end{array}$ & At day 14 with ND+IB & This study \\
\hline
\end{tabular}

ND, Newcastle disease; IB, Infectious bronchitis. 
TABLE 2 | Primers used to amplify the complete genome of lineage 5 NDV.

\begin{tabular}{lcll}
\hline Fragment & Location & Forward primer sequence $\left(\mathbf{5}^{\prime} \boldsymbol{\rightarrow} \mathbf{3}^{\prime}\right)$ & Reverse primer sequence $\left.\mathbf{( 5}^{\prime} \boldsymbol{\rightarrow} \mathbf{3}^{\prime}\right)$ \\
\hline 1 & $1-2792$ & ACCAAACAGAGAATCTGTGAGGTAC & TCGGGCTACTGCTCGGAGAT \\
2 & $2541-5926$ & GTCCAGCTACCTGCCGACTT & GCCTGTCACGATGACTTGAGA \\
$3^{*}$ & $5673-9156$ & CTGAAGGCGCACTCACTACG & AGATGTGCCGCCGTAGAAGAT \\
& $4258-8657$ & CCTGCGGAGTGTGAAAGTCA & GGAGCACTCCGGTATCTTGG \\
5 & $8644-12,183$ & AACCGGAGTGCTCCATCCC & AGATTCCCAGCTGTGGGCAG \\
5 & $11,745-15,192$ & CCCGGAACAGAAGCTGGTCA & ACCAAACAGAGATTGGTGAACGAC \\
\hline
\end{tabular}

*An additional primer was designed for fragment 3 which was located in the hypervariable region of the genome. Primer locations are based on NDV strain chicken/Banjarmasin/010/10 (HQ697254.1).

\section{Synthesis of Double Strand DNA}

Based on the manufacturer's instructions of using KAPA HiFi Hot Start Ready Mix (KAPA Biosystems, USA) kit, the cDNA was used to synthesize a double strand DNA. Briefly, $3 \mu \mathrm{L}$ of cDNA was combined with $12.5 \mu \mathrm{L}$ of $2 \times$ KAPA HiFi Hot Start Ready Mix, $0.9 \mu \mathrm{L}$ of $10 \mu \mathrm{M}$ forward primer and reverse primer up to $25 \mu \mathrm{L}$ volume with PCR-grade water. The thermal cycling protocol for the PCR was included with an initial denaturation at $95^{\circ} \mathrm{C}$ for $3 \mathrm{~min}$ followed by a sequence of 30 cycles, which comprised denaturation at $98^{\circ} \mathrm{C}$ for $20 \mathrm{~s}$, annealing at $60^{\circ} \mathrm{C}$ for $20 \mathrm{~s}$, extension at $72^{\circ} \mathrm{C}$ for $2 \mathrm{~min}$ and final extension hold at $72^{\circ} \mathrm{C}$ for $5 \mathrm{~min}$. The PCR product was detected in $0.8 \%$ agarose gel electrophoresis and purified using MEGAquickspin ${ }^{\mathrm{TM}}$ Total Fragment DNA Purification Kit (iNtRON Biotechnology, South Korea) as per manufacturer's instructions.

\section{Next-Generation Sequencing Library Preparation and Sequencing}

Each DNA originated from the isolates was quantified by using Qubit dsDNA HS Assay Kit (Invitrogen, USA) and then normalized to $0.2 \mathrm{ng} / \mu \mathrm{L}$. A total of $1 \mathrm{ng}$ of DNA was subjected to library preparation using Nextera XT DNA Sample Prep Kit (Illumina Inc., San Diego, CA, USA) by following the manufacturer protocol. Briefly, the DNA was tagmented (fragmented and tagged) by the Nextera XT transposome. The tagmented DNA was used as template in a $50 \mu \mathrm{l}$ of PCR with 12 cycles and processed as outlined in the Nextera XT protocol. Additionally, AMPureXP beads (Beckman Coulter Inc., Fullerton, CA, USA) was applied to purify the amplified DNA.

After PCR clean up, DNA fragment size and library concentration was analyzed by using 2100 Bioanalyzer (Agilent Technologies, USA) and Library Quantification Kit (KAPA Biosystems, USA). Afterward, DNA libraries were normalized to $4 \mathrm{nM}$ and libraries with unique indexes were pooled in equal volumes. Pooled libraries were denatured and diluted with $0.2 \mathrm{~N}$ $\mathrm{NaOH}$ and pre-chilled Hybridization Buffer (HT1) to produce a denatured $12 \mathrm{pM}$ library in $1 \mathrm{mM} \mathrm{NaOH}$ solution. The final library was sequenced using MiSeq (Illumina Inc., San Diego, CA, USA) with the read length of $2 \times 150 \mathrm{bp}$.

\section{Analysis of the Data}

The overlapping paired-end reads were filtered on the Phred quality score (Q30) and imported to CLC Genomics Workbench software version 7.5.1 (CLC bio, Aarhus, Denmark) for adapter trimming and de novo assembly of the paired-end reads to contigs. The contigs were then subjected to BLASTN at NCBI and based on the highest sequence similarity and lowest $E$-value, reference genomes were determined. Low coverage contigs were excluded and when necessary, partial but overlapping contigs were combined. Final consensus having more than $99 \%$ coverage to full genome sequences were then examined for appropriate assembly depending on the length and the presence of the expected intact NDV open reading frames.

\section{Confirmation Using Sanger Sequencing}

Two gaps were observed in the consensus of IBS005/11 at the nucleotide positions of 1708-1743 and 1821-1882. Forward primer sequence $5^{\prime}$-GCCATCCCAAGACAACGACA- 3 ' aligning nucleotide position of consensus at 1555 and the reverse primer sequence $5^{\prime}$ - CCCTGGGCCGTTATTATGCT-3' aligning position of IBS005/11 consensus at 1956 were designed by using NCBI Primer 3 software to close the gap. The PCR product was observed in $1.5 \%$ agarose gel. Similarly, 7 nucleotides gap was observed in the consensus of MB128/04 at the nucleotide positions 1698-1708. This gap was closed using the same forward and the reverse primer sequences earlier mentioned. Additionally, the recombination region (1-2766 bp) including the 6 nucleotides deletion at the position 1647 of $5^{\prime}$ NCR of the NP gene was validated twice by sequencing the fragment 1 (Table 2) of the IBS025/13 NDV isolate by primer walking technology.

The cDNA template was synthesized as previously described and PCR was performed according to PCR conditions mentioned above. Both PCR products were purified with MEGAquickspin ${ }^{\mathrm{TM}}$ Total Fragment DNA Purification Kit (iNtRON Biotechnology, South Korea) as per manufacturer's instructions. Sanger sequencing was performed from both directions in order to close the gaps and confirmation of recombination region in the IBS 025/13 isolate. Finally, the obtained sequences were aligned with the consensus by using MEGA6 software (Tamura et al., 2013).

\section{Amplification of the $3^{\prime}$ - and $5^{\prime}$ - Terminal Ends of the Viral RNA}

To determine the leader and trailer at the $3^{\prime}$ - and $5^{\prime}$ terminal ends of the viral RNA, rapid amplification of 
cDNA ends (RACE) was performed as described earlier on de Leeuw and Peeters (1999). The $5^{\prime}$ end primer L-cDNA (5' - AAGTCACAATACTGGGTCTCAG - $\left.3^{\prime}\right)$, which was designed from $\mathrm{L}$ gene of genotype VII NDV strain, chicken/Banjarmasin/010/10 (HQ697254.1) was used to generate single-stranded cDNA as described above. The T4 RNA ligase (New England Biolabs Inc, USA) was then used to ligate the generated single-stranded cDNA so as to anchor primer $\left(5^{\prime}-\right.$ CACGAATTCACTATCGATTCTGGATCCTTC $\left.-3^{\prime}\right)$. One micro liter of ligation mixture was used for PCR with KAPA HiFiHotStartReadyMix (KAPA Biosystems, USA) in accordance with the manufacturer's instructions. The primers used were anchor-complementary (5'-GGATCCAGAATCGATAGTGAATTCG- $3^{\prime}$ ) and L-PCR (5'-CAGCCAAGGGATATTACAGTAACT- $3^{\prime}$ ). The PCR consisted of 30 cycles of $1 \mathrm{~min}$ at $98^{\circ} \mathrm{C}, 20 \mathrm{~s}$ at $60^{\circ} \mathrm{C}$ and $20 \mathrm{~s}$ at $72^{\circ} \mathrm{C}$. The PCR products were then cloned into pJET 1.2 blunt/cloning vectors (Thermo Fisher Scientific, Inc, USA) and verified by sequencing. The anchor primer was ligated to the $3^{\prime}$ prime end of genomic RNA with T4 RNA ligase as described above in order to determine the sequence of the $3^{\prime}$-terminal ends. Then, the anchor-complementary primer was used to generate the cDNA. Hence, anchor-complementary and NP-PCR primers were used to conduct PCR. Primer (5'-GGAGCTGCTCGTATTCGTC-3') was used to generate fragments for strains MB076/05, IBS002/11, IBS005/11, and MB128/04. Meanwhile, NP-PCR primer ( $5^{\prime}-$ CGAGGAGCTGTTCGTACTCATCAA-3') was used for strain IBS025/13. The PCR conditions were the same as have been described above.

\section{Recombination among NDV Sequences}

SimPlot program (Ray, 2003) was used to identify putative recombination breakpoints in the sequenced whole genome of NDV isolates and to identify sequences possibly originated from a recombination. The program is based on a sliding window process and consists of a way of graphically displaying the coherence of the sequence relationship over the entire length of a set of aligned homologous sequences. The window width and the step size were set to $200 \mathrm{bp}$ (or $500 \mathrm{bp}$ ) and $20 \mathrm{bp}$, respectively. In addition, Recombinant Program v4.56 (RDP4) was used for the detection of recombination events, likely parental isolates of recombinants and recombinant breakpoints. Various methods such as RDP, GENECONV, Chimaera, MaxChi, BOOTSCAN, and SISCAN with default settings (Martin et al., 2005) were used by the RDP4 program.

\section{Phylogenetic and Pairwise Sequence Comparisons (PASC) Analysis}

The nomenclature of the NDV isolates in this study was based on genotypes (Ballagi-Pordány et al., 1996; Czeglédi et al., 2006) and lineages (Aldous et al., 2003; Snoeck et al., 2009; Munir et al., 2012). CLUSTALW (Thompson et al., 1997) was used to align all the NDV sequences while MEGA6 software was used to analyze all the phylogenetic. The model with lowest BIC (Bayesian Information Criterion) value was selected as the most suitable model for phylogenetic analysis (Tamura et al., 2013). The reliability of the lineages defined for NDV was determined by Pair-Wise Sequence Comparisons (PASC) using selected NDV strains available in GenBank representing all lineages except lineage 6 and Malaysian isolates. All reference isolates used in this study were named first with accession number followed by country of origin, respected lineage and year of isolation. Also, mean distances among and within lineages were calculated using PASC in MEGA6 software.

\section{Ethics Statement}

The animals used for this research were kept in the house and animal care procedures were conducted according to the local animal welfare regulations and EU directive (EU Directive on the protection of animals used for scientific purposes 2010/63/EU) (EU, 2010) under bio-safety level (BSL2) enhanced experimental animal facility at the Faculty of Veterinary Medicine, UPM. The animal experimental protocol for Intracerebral Pathogenicity Index (ICPI) study was approved by the Institutional of Animal Care and Use Committee (IACUC), Faculty of Veterinary Medicine, UPM (reference number UPM/IACUC/AUP-R028/2013); the local animal care authority. Animals were monitored for a minimum of three times per day by a qualified and registered veterinarian to ensure animal welfare and health. The end point of all these in vivo animal experiments was death; although, a humane endpoint was pre-defined in the protocol and applied to prevent any pain, distress or suffering. The humane endpoint was decided for chicks used for ICPI study manifested terminal clinical signs. Animals showing terminal signs including anorexia and paralysis were sacrificed by cervical dislocation under sedation in accordance with standard guidelines (EU, 2010; Leary et al., 2013).

\section{Intracerebral Pathogenicity Index Assessment in SPF Chickens}

Pathogenicity of NDV isolates was assessed by using a standard ICPI test as earlier stated (OIE, 2012). Briefly, $50 \mu \mathrm{l}$ of allantoic fluid of each NDV isolate with an HA titer more than $16 \mathrm{HA}$ units $/ 50 \mu \mathrm{l}$ and diluted 10 -fold in PBS without antibiotics were inoculated intracerebrally to 1 -day-old chicks $(n=10)$. Ten chicks were kept as uninoculated control. All the chicks were being monitored at least three times per day for an 8-day period of observation. The NDV isolates that scored an ICPI $>1.5$ were identified as velogenic strains, $<0.7$ as lentogenic and those with intermediate ICPI values were considered as mesogenic strains (OIE, 2012).

\section{RESULTS}

\section{Whole Genome Sequencing of NDV}

The present study proposes a pipeline to generate consensus level sequences with more than $99 \%$ coverage of the full genome. The consensus level genome sequences of five NDV isolates belonging to genotype VII were generated for the first time in Malaysia. The whole genome sequences of these NDV isolates were deposited in the GenBank and are available under the following accession numbers: KR074407 for MB128/04, KR074406 for 
TABLE 3 | Summary of the genome organization and the predicted protein length of the sequenced MB128/04, MB076/05, IBS002/11, and IBS005/11 isolates.

\begin{tabular}{|c|c|c|c|c|c|c|c|c|}
\hline Gene & $\begin{array}{c}\text { Gene start } \\
\text { position (nt) }\end{array}$ & $\begin{array}{c}\text { 3'UTR } \\
\text { length (nt) }\end{array}$ & $\begin{array}{l}\text { Coding sequence } \\
\text { positions (nt) }\end{array}$ & $\begin{array}{c}\text { 5'UTR } \\
\text { length (nt) }\end{array}$ & $\begin{array}{l}\text { Gene end } \\
\text { position (nt) }\end{array}$ & $\begin{array}{l}\text { Intergenic region } \\
\text { length (nt) }\end{array}$ & $\begin{array}{c}\text { Gene } \\
\text { length (nt) }\end{array}$ & $\begin{array}{c}\text { Protein } \\
\text { length (aa) }\end{array}$ \\
\hline NP & $56-65$ & 66 & $122-1591$ & 217 & 1799-1808 & 1 & 1753 & 489 \\
\hline$P$ & 1810-1819 & 83 & 1893-3080 & 180 & $3251-3260$ & 1 & 1451 & 395 \\
\hline M & $3262-3271$ & 34 & $3296-4390$ & 112 & 4493-4502 & 1 & 1241 & 364 \\
\hline $\mathrm{F}$ & $4504-4513$ & 46 & $4550-6211$ & 84 & 6284-6295 & 31 & 1792 & 553 \\
\hline $\mathrm{HN}$ & $6327-6336$ & 91 & 6418-8133 & 195 & 8319-8328 & 47 & 2002 & 571 \\
\hline L & $8376-8385$ & 11 & $8387-15,001$ & 77 & $15,069-15,078$ & - & 6703 & 2204 \\
\hline
\end{tabular}

UTR, untranslated region.

TABLE 4 | Summary of the genome organization and predicted protein lengths in the sequenced IBS025/13 isolate.

\begin{tabular}{|c|c|c|c|c|c|c|c|c|}
\hline Gene & $\begin{array}{l}\text { Gene start } \\
\text { position (nt) }\end{array}$ & $\begin{array}{c}3^{\prime} \text { UTR } \\
\text { length (nt) }\end{array}$ & $\begin{array}{l}\text { Coding sequence } \\
\text { positions }\end{array}$ & $\begin{array}{c}5^{\prime} \text { UTR } \\
\text { length (nt) }\end{array}$ & $\begin{array}{c}\text { Gene end } \\
\text { position (nt) }\end{array}$ & $\begin{array}{l}\text { Intergenic region } \\
\text { length (nt) }\end{array}$ & $\begin{array}{c}\text { Gene } \\
\text { length (nt) }\end{array}$ & $\begin{array}{c}\text { Protein } \\
\text { length (aa) }\end{array}$ \\
\hline NP & $56-65$ & 66 & $122-1591$ & 210 & $1791-1801$ & 2 & 1746 & 489 \\
\hline$P$ & $1804-1813$ & 83 & 1887-3074 & 180 & $3245-3254$ & 1 & 1451 & 395 \\
\hline M & 3256-3265 & 34 & $3290-4384$ & 112 & $4487-4496$ & 1 & 1241 & 364 \\
\hline$F$ & 4498-4507 & 46 & 4544-6205 & 84 & 6280-6289 & 31 & 1792 & 553 \\
\hline $\mathrm{HN}$ & $6321-6330$ & 91 & $6412-8127$ & 195 & 8313-8322 & 47 & 2002 & 571 \\
\hline $\mathrm{L}$ & 8370-8379 & 11 & $8381-14,995$ & 77 & $15,063-15,072$ & - & 6703 & 2204 \\
\hline
\end{tabular}

UTR, untranslated region.

MB076/05, KR074404 for IBS002/11, KR074405 for IBS005/11 and IBS025/13 for KT355595.

Summary of the genomic features of the Malaysian isolates, including starting and ending positions of each gene, intergenic, and coding regions, represented in Tables 3, 4. The genome length of $4 \mathrm{NDV}$ isolates (MB128/04, MB076/05, IBS002/11, and IBS005/11) and IBS025/13 isolate was calculated to be 15,192 nts and 15,186 nts respectively, which putatively consisted of 6 different genes with each gene containing a single or multiple open reading frames (ORFs) as indicated in Tables 3, 4.

\section{Biological and Molecular Pathogenicity Assessment}

The isolates in the current study exhibited ICPI values more than 1.5 and the $\mathrm{F}$ protein cleavage site sequences exhibited ${ }^{112} \mathrm{R} / \mathrm{K}-$ $\mathrm{R}-\mathrm{Q}-\mathrm{R} / \mathrm{K}-\mathrm{R} \downarrow \mathrm{F}^{117}$ motif similar to mesogenic and velogenic NDV strains, which is another characteristic considered by the World Organization for Animal Health (OIE) to assess the pathogenicity (OIE, 2012) (Table 5).

\section{Phylogenetic and Pairwise Sequence Comparison (PASC) Analysis of NDV Isolates}

Genomic information is the basis used by the two currently available methods in NDV classification, where one is to identify strains by lineages and the other by classes/genotypes. The phylogenetic and pairwise sequence comparison data presented in this study is based on the lineage classification proposed by Aldous et al. (2003) and Munir et al. (2012). According to the
TABLE 5 | Biological and molecular characterization of NDV isolates.

\begin{tabular}{lcc}
\hline NDV isolate & F cleavage site & ICPI \\
\hline MB128/04 & $112 R R Q K R \downarrow F^{117}$ & 1.68 \\
MB076/05 & $112 R R Q K R \downarrow F^{117}$ & 1.84 \\
IBS002/11 & $112 R R R K R \downarrow F^{117}$ & 1.76 \\
IBS005/11 & $112 R R R K R \downarrow F^{117}$ & 1.71 \\
IBS025/13 & $112 R R Q K R \downarrow F^{117}$ & 1.63 \\
\hline
\end{tabular}

phylogenetic analysis of partial $\mathrm{F}$ gene, all the isolates clustered with lineage 5 (Figure 1). Moreover, in line with the PASC analysis, the isolates also have the highest percentage identity (PI) to NDV strains belonging to lineage 5. The NDV isolates MB128/04 and MB076/05 isolated in 2004 and 2005, respectively, showed the highest PI of $92.41 \%$ to lineage 5 (Table 6). Meanwhile, PASC analysis of the genetic similarity among sublineage under lineage 5 indicated that the isolates showed the highest PI to sub-lineage 5a. However, except for IBS025/13, the genomic divergence between the lineage $5 \mathrm{a}$ and the sequenced viruses isolated during the period from 2004 to 2011 is increasing (Table 7). The phylogenetic tree was drawn based on nucleotide sequence of complete genome sequences, and it shows the clustering of the isolates with lineage 5 (Figure 2A). The robustness of the genetic groupings and topology of the phylogenetic trees were also confirmed by the findings obtained from the PASC analysis of the complete genome as well as F, HN, 


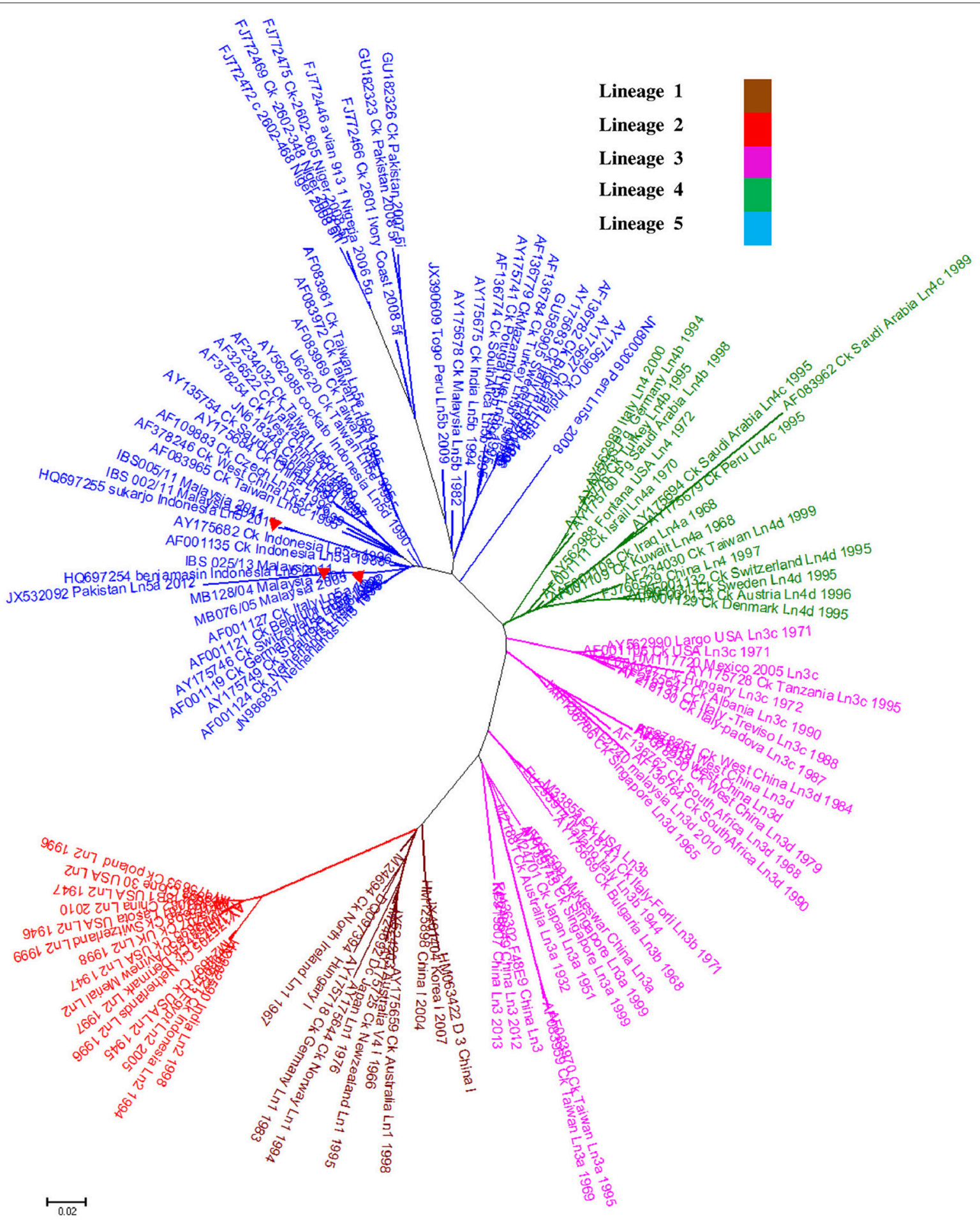

FIGURE 1 | A phylogenetic analysis of the Newcastle disease viruses; Phylogenetic relationship among 120 published NDV isolates and 5 Malaysian isolates based on $\mathrm{F}$ gene nucleotide sequences between $\mathbf{4 7}$ and $\mathbf{4 2 2}$ positions. The sequences used were obtained from GenBank. As identified by Aldous et al. (2003), Class II viruses representing lineages 1-5 $(n=120)$ and Malaysian isolates from 2004 to $2013(n=5)$ identified with 373 base pair region encoding the amino terminal end of the fusion protein. Tree construction was done using the Neighbor-Joining method with the maximum composite likelihood substitution model after 1000 bootstrap replication by using MEGA 6. Lineage 6 of APMV-1 was excluded in the analysis. Isolates presented in this study was marked with a red triangle $(\mathbf{\Lambda})$. 
TABLE 6 | Pairwise sequence comparison of the partial $F$ gene sequences of Malaysian isolates based on different lineages.

\begin{tabular}{lccccc}
\hline NDV Isolate & \multicolumn{5}{c}{ Percentage identity (\%) } \\
\cline { 2 - 6 } & $\mathbf{1}$ & $\mathbf{2}$ & $\mathbf{3}$ & $\mathbf{4}$ & $\mathbf{5}$ \\
\hline MB128/04 & 83.74 & 81.16 & 87.42 & 89.09 & 92.41 \\
MB076/05 & 83.74 & 81.16 & 87.42 & 89.09 & 92.41 \\
IBS002/11 & 81.48 & 79.84 & 84.65 & 85.81 & 88.42 \\
IBS005/11 & 81.48 & 79.84 & 84.65 & 85.81 & 88.42 \\
IBS025/13 & 83.57 & 80.66 & 86.50 & 87.87 & 91.32
\end{tabular}

TABLE 7 | PASC analysis of the partial F gene sequences of Malaysian isolates based on lineage 5 .

\begin{tabular}{lccccccccc}
\hline NDV isolate & \multicolumn{8}{c}{ Percentage identity (\%) } \\
\cline { 2 - 9 } & $\mathbf{5 a}$ & $\mathbf{5 b}$ & $\mathbf{5 c}$ & $\mathbf{5 d}$ & $\mathbf{5 e}$ & $\mathbf{5 f}$ & $\mathbf{5 g}$ & $\mathbf{5 h}$ & $\mathbf{5 i}$ \\
\hline MB128/04 & 96.22 & 92.23 & 93.74 & 93.51 & 93.16 & 85.79 & 86.33 & 83.56 & 86.33 \\
MB076/05 & 96.22 & 92.23 & 93.74 & 93.51 & 93.16 & 85.79 & 86.33 & 83.56 & 86.33 \\
IBS002/11 & 91.51 & 87.57 & 90.88 & 90.03 & 88.67 & 81.77 & 82.31 & 79.54 & 83.51 \\
IBS005/11 & 91.51 & 87.57 & 90.88 & 90.03 & 88.67 & 81.77 & 82.31 & 79.54 & 83.51 \\
IBS025/13 & 94.82 & 91.37 & 92.58 & 92.6 & 91.89 & 84.45 & 84.46 & 81.68 & 85.66
\end{tabular}

$\mathrm{M}$, and L protein sequences of MB128/04, MB076/05, IBS002/11 and IBS005/11 isolates, respectively, whilst PASC analysis of NP and $\mathrm{P}$ protein sequences of IBS025/13 isolate showed the highest percentage identity to lineage 2 viruses (Table 8). Furthermore, maximum likelihood phylogenetic trees constructed based on NP and $\mathrm{P}$ proteins of IBS025/13 isolate indicated that those proteins were clustered with lineage 2 viruses and substantiated the results obtained from PASC analysis (Figure 2B).

\section{Recombination Analysis of NDVs Using Simplot and RDP}

A standard similarity plot (Simplot) (Ray, 2003) was used to analyze the possible events of recombination in the sequenced isolates with representative isolates of lineages 1-5 obtained from GenBank. Since the analysis revealed that NP and P gene regions of isolate IBS025/13 have the highest genomic similarity toward lineage 2 viruses over lineage 5 (Figures 3A,B), we also investigated the first $1 / 3$ of NDV genome alignment to identify potential breakpoints by using recombinant detection program (RDP) (Martin et al., 2005). Possible breakpoints at nucleotide position 1-2766 and 2767-2984 were detected by $p$-value equal to RDP (8.418E-4), Geneconv (1.617E-3), BootScan (8.349E-4), MaxChi (1.612E-1) and SiScan (2.509E-10). The most convincing evolutionary evidence for recombination was the occurrence of incongruent phylogenetic trees (Zhang et al., 2010). Maximum composite likelihood phylogenic trees were constructed in both break points using MEGA 6 software with 1000 bootstrap values according to the best model selected on lowest BIC. The results confirmed clustering of IBS025/13 isolate with lineage 2 NDVs at breakpoint 1-2766 and breakpoint 2767-2984 with lineage 5 NDVs (Figures 4A,B). Further evidence on the event of recombination in NDV was provided by sequence comparisons within the species-defining clusters (Han et al., 2008). Nucleotide fragment of 1-2766 of IBS025/13 was used as a query in BLAST in the GenBank, which showed the highest sequence identity of $99 \%$ to Lasota (AF077761), B1 (AF309418), Clone 30 (Y18898) lineage $2 \mathrm{NDV}$ vaccine strains (data not shown). Sequence analysis confirmed the NP gene of IBS025/13 from nucleotide position 55-1801, which include both the $3^{\prime}$ and $5^{\prime}$ untranslated regions (UTR) were derived from lineage 2 isolates (Table 4). In addition, the deletion of $6 \mathrm{nts}$ at the UTR region of NP gene revealed similar genome length to lineage $2 \mathrm{NP}$ genes. Meanwhile, the entire $3^{\prime}$ UTR of P gene (1804-1886) and two-thirds of P gene from position $1887-2776$ of IBS025/13 also resemble lineage 2 isolates (Table 4).

\section{DISCUSSION}

In 2013, more than 3000 ND outbreaks were reported from Asian countries to the World Organization for Animal Health (WAHID, 2015). Characterizing and understanding the molecular epidemiology of the currently circulating NDV strains in the world, is essential for the controlling and preventing ND outbreaks (Miller et al., 2010). The current approach used only five sets of primers to cover the whole genome of NDV, and the protocol could support the multiplexing of 94 samples by tagging unique combination of indexes during a single sequencing run with a fast turn-around time, which dramatically reduced the cost and laborious work needed to be conducted in complete genome sequencing. Since adopted method used a unique combination of indexes to each NDV sample during multiplexing and raw sequenced data was filtered according to the tagged indexes, generated sequences are originated purely from the NDV isolates obtained from outbreaks. Moreover, NGS based on Ion Torrent Personal Genome Machine (PGM, Life technologies), has been used to sequence the complete genome of avian paramyxoviruses type 4 (Wang et al., 2013).

Genome lengths of the MB128/04, MB076/05, IBS002/11 and IBD005/11 isolates were similar to the genome length of genotype V-VIII. and IX in class II APMV-1. Studies have shown that the genome length of these genotypes have evolved by an insertion of $6 \mathrm{nts}$ at the nucleotide position of 1647 in the $5^{\prime}$ non-coding region (NCR) of the NP gene of early genotypes (I-IV), which have genome length of 15,186 nts (Czeglédi et al., 2006). Interestingly, genome length of IBS025/13 NDV isolated in 2013 was 15,186 nts, similar to the genome length of early NDV genotypes (I-IV) isolated during 19301960s (Czeglédi et al., 2006). In this isolate, deletion of 6 nts at the same position of $5^{\prime} \mathrm{NCR}$ of the NP gene was observed subsequently confirmed by targeted region amplification and sequencing using primer walking technology. In addition, the NP and P gene regions of IBS025/13 were sequenced thrice using Sanger sequencing to confirm the recombinant events.

The OIE recognizes the ICPI as a test which can be used to assess the pathogenicity of NDV. An NDV strain with an ICPI $\geq 0.7$ is identified as a virulent or "notifiable" to the 


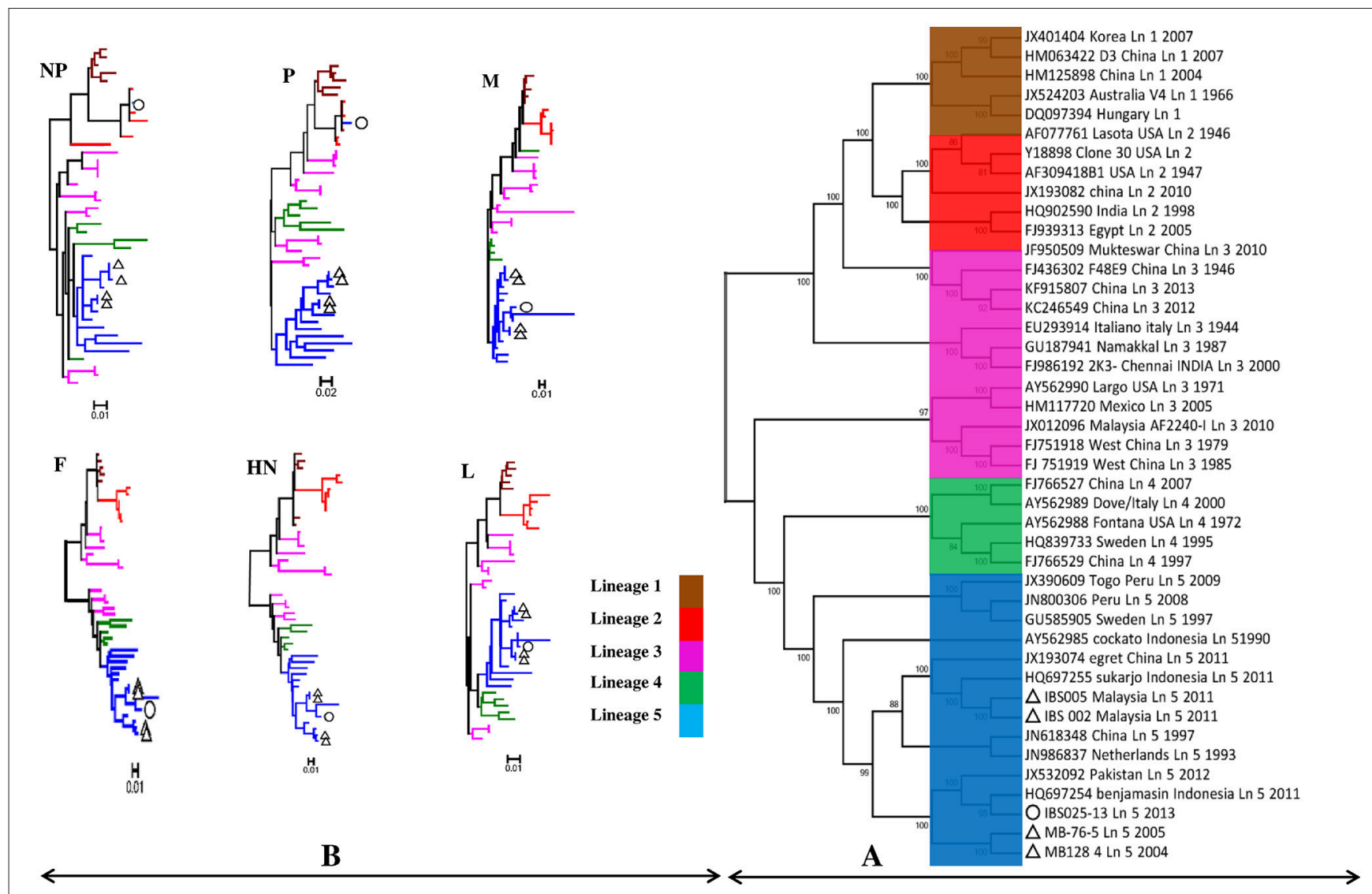

FIGURE 2 | The maximum likelihood phylogenetic trees constructed by using $\mathbf{5}$ Malaysian NDV isolates. (A) The phylogenetic tree based on nucleotide sequence of complete genome compared with 38 representative complete genomes of lineage 1-5 belongs to APMV-1. (B) The phylogenetic trees based on amino acids sequence of NP, P, M, F, HN, and L proteins compared with 38 representative protein sequence of lineage 1-5 belongs to APMV-1. The tree is drawn to scale, with branch lengths measured in the number of substitutions per site. Evolutionary analyses were conducted in MEGA6. Malaysian isolates MB128/04, MB076/05, IBS002/11, and IBS005/11 presented in this study are marked with a triangle $(\Delta)$ and IBSO25/13 with a circle (o).

OIE. The cleavage site motif ${ }^{112} \mathrm{R} / \mathrm{K}-\mathrm{R}-\mathrm{Q}-\mathrm{R} / \mathrm{K}-\mathrm{R} \downarrow \mathrm{F}^{117}$ is cleavable by a wide range of proteases that subsequently cause systemic infection (Panda et al., 2004; Maminiaina et al., 2010; Miller et al., 2010). The cleavage site motif of the isolates also demonstrated the sequences of velogenic strains and these findings confirm the velogenic nature of the Malaysian NDV isolates (Table 5) reported in this study.

Previously, Munir et al. (2012) have indicated that NDV sublineages under lineage 5 can be identified with cut-off PI at 95\% based on partial $F$ gene sequences. In that study, viruses in lineage 5 can be further divided into 9 sub-lineages, from 5 a to $5 \mathrm{i}$ with previously characterized NDV isolates including recent isolates reported from African countries and Pakistan. Based on the above study, MB128/04 and MB076/05 can be placed in the sublineage $5 \mathrm{a}$. One of the salient findings of this study is, although IBS002/11 and IBS005/11 that were isolated in 2011 have the highest PI-value to the sub-lineage $5 \mathrm{a}$, the PI-value was $<95 \%$. The findings illustrate the evolutionary pattern observed in NDV isolates from 2004 to 2011 and urged a reviewing of the current sub-lineage cut off point of lineage 5 in NDV classification or placing NDV isolates IBS002/11 and IBS005/11 under a new sub-lineage of lineage 5. Furthermore, the findings of this study support a reviewing of the current nomenclature of NDV as proposed by Miller et al. (2010) and Munir et al. (2012). The phylogenetic tree drawn based on complete nucleotide sequences of isolates shows the clustering of the isolates with lineage 5 and these findings agreed with Miller et al. (2010), who propounded that currently, the predominant circulating NDV isolates in the Asian region are of lineage 5.

In comparison to the traditional NDV classification method by partial $\mathrm{F}$ gene, the whole genome analysis is advantageous for the identification of recombination events. Other previous studies have reported the evidence of recombination in $\mathrm{F}$ gene sequences between genotype II and genotype VII, poultry and ostrich NDVs (Yin et al., 2011). Moreover, Chong et al. (2010) and Han et al. (2008) have reported recombinants of NDVs that involved multiple genotypes. The results of the current study present the first natural recombination event detected between lineage 2 and 5 following isolation and sequencing of virus from an ND outbreak in chicken.

Vaccination against NDV has been described as the most effective prevention strategy seconded to strict bio-security 
TABLE 8 | Estimates of the evolutionary distance of NP, P, F, HN, M, L amino acid and complete genome nucleotides sequences between Malaysian NDV isolates and each lineage.

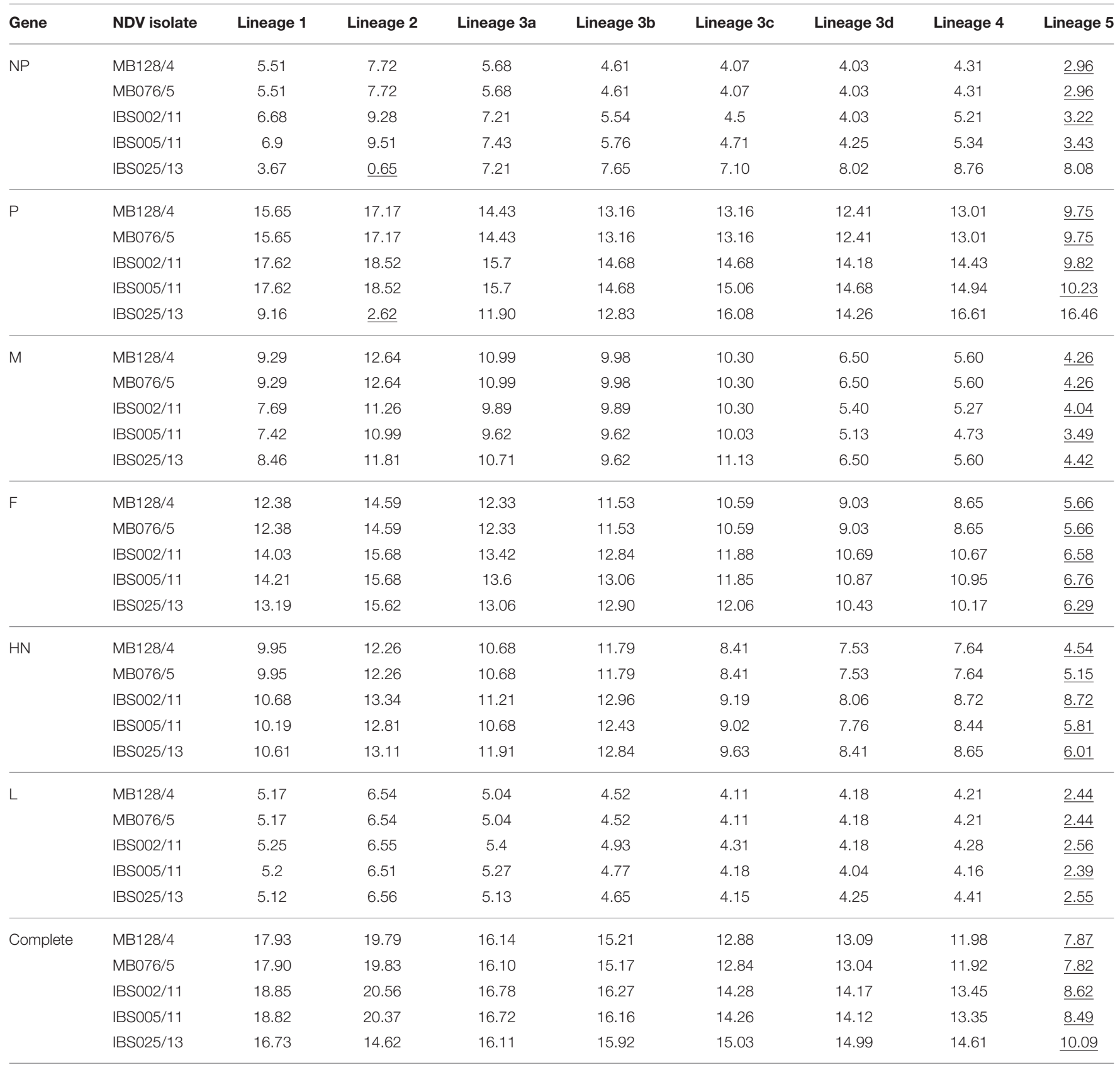

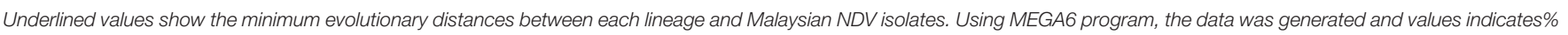
nucleotide and amino acids distance respectively.

measures in modern poultry farming where live and killed vaccines are widely used throughout various countries in the world. Despite intensive vaccination, the occurrence of NDV outbreaks in endemic countries in Asia, Africa, and Central America is puzzling (Czeglédi et al., 2006). High-density rearing in modern poultry farming, which enhances close animal-to-animal contact, favoring transmission of virulent viruses over milder forms and selective immune pressure exerted by improper vaccination, can affect the evolution of circulating virulent viruses (Miller et al., 2010; Zenglei and Liu, 2015). Additionally, improper vaccination strategy, immune suppression and presence of variant NDV strains have been implicated as the main underlying factors of poor vaccine efficacy against ND (Okoye and Shoyinka, 1983; Yu et al., 2001; Cho et al., 2007; Dortmans et al., 2012; Perozo et al., 2012). 

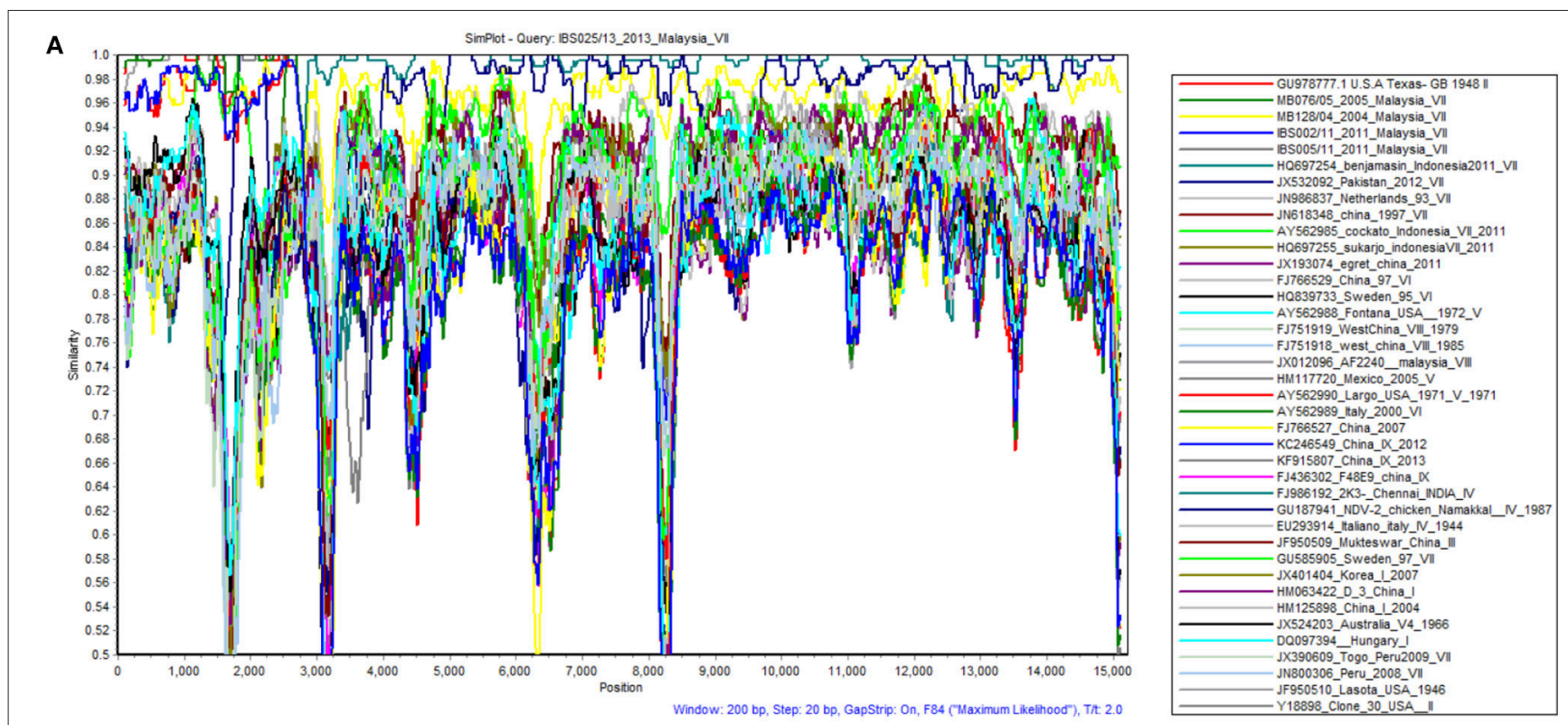

B
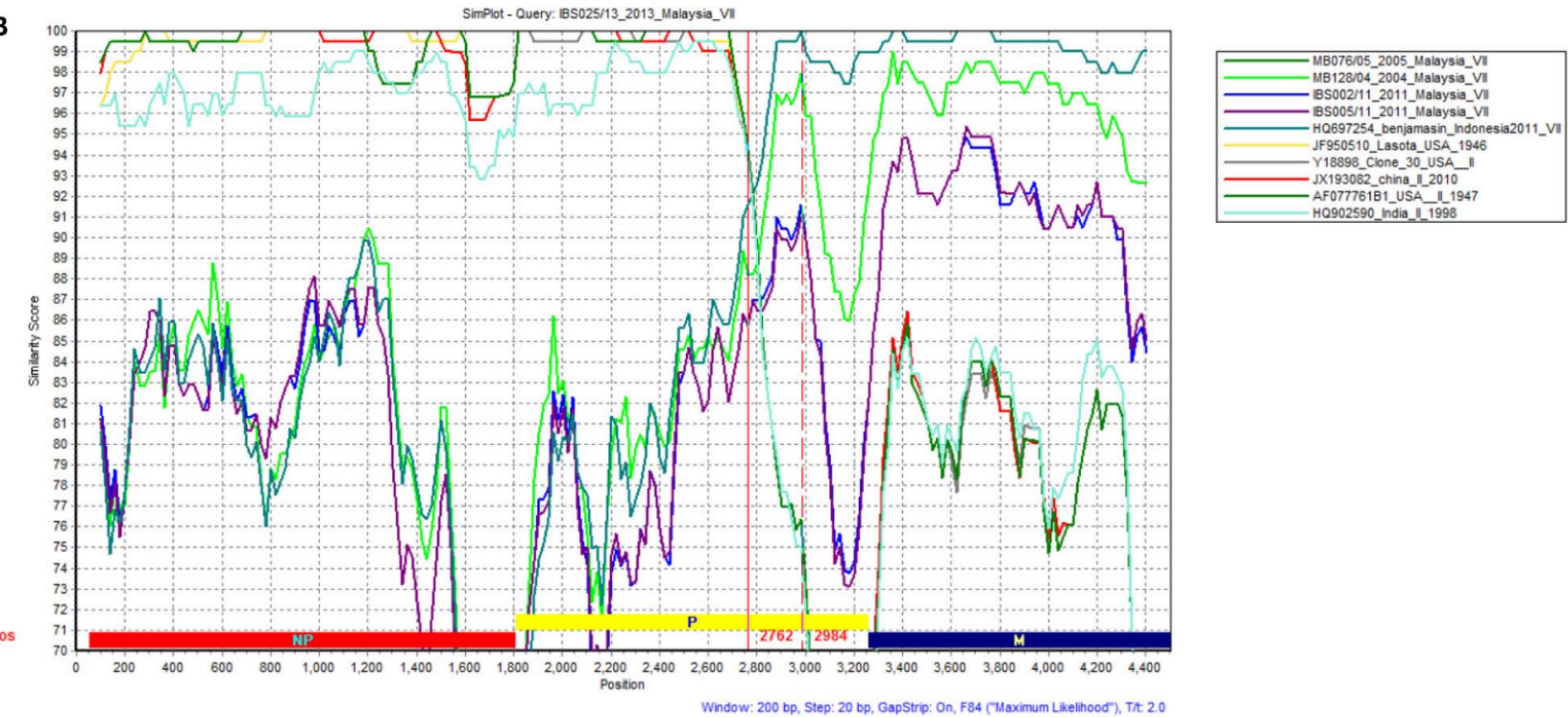

FIGURE 3 | Results from SimPlot Analysis of IBS025/13. The evidence for recombination of strain IBS025/13. (A) The similarity of the complete genome of IBS025/13 from SimPlot analysis with 38 representative complete genomes belonged to APMV-1. Standard similarity plot constructed using all sites of the complete genome within a sliding window of $200 \mathrm{bp}$ wide centered on the position plotted with a step size between plots of $20 \mathrm{bp}$. The $\mathrm{y}$-axis gives the percentage of identity. (B) The similarity of the first 1/3 genome representing NP, P, and M gene of IBS025/13 from SimPlot analysis with most similar nucleotide sequences belong to APMV-1. Standard similarity plot constructed using all sites of the first $1 / 3$ genome with a window size of 200 bp and a step size of 20 bp. The $y$-axis gives the percentage of identity. Red vertical lines indicate the possible breakpoints.

Based on bioinformatics analysis, it has been reported that vaccination with live attenuated viruses altered the evolution of NDV by sustaining a large effective population size of a vaccinerelated genotype, allowing for co-infection and recombination of vaccine and wild-type strains (Chong et al., 2010). In that study, recombination events in NP gene of Cockatoo/14698 Indonesia (AY562985) and F gene of Layer/SRZ03 China (EU167540) of genotype VII isolate with genotype I isolates were identified by maximum likelihood trees and RDP3 program (Chong et al., 2010). The current study provides the evidence of recombination in NDV between vaccine lineage and the circulating lineage 5 following an isolation and sequencing. Since live attenuated vaccines are being carried out in most of the NDV-endemic countries, the likelihood of recombination between vaccine and field strains is relatively high. The magnitude of occurrence and underlying molecular mechanisms of natural recombination 


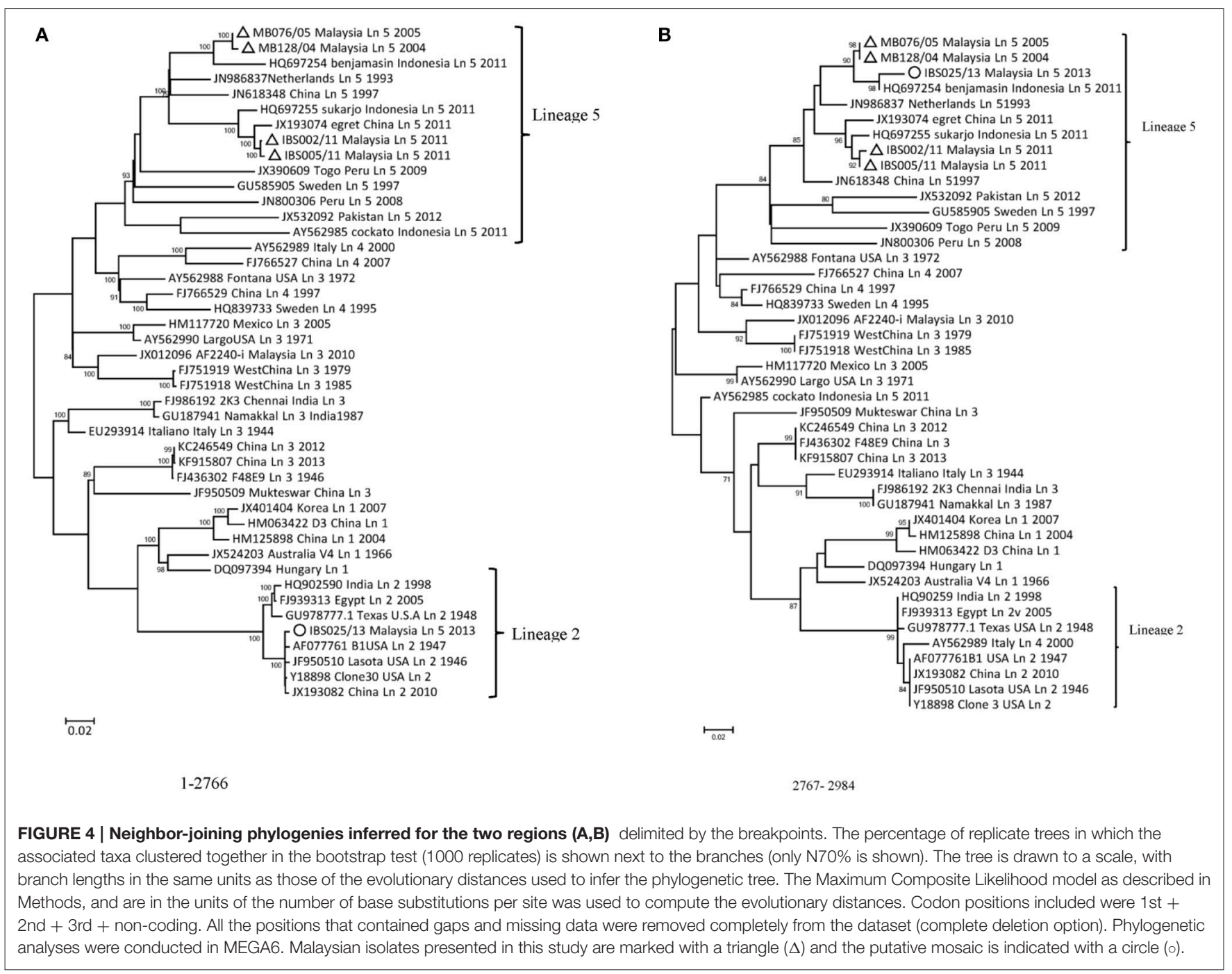

in NDV is not well documented. Many research groups have documented natural recombination events for NDV (Han et al., 2008; Qin et al., 2008; Chong et al., 2010; Zhang et al., 2010; Yin et al., 2011). However, it has also been suggested that the recombination between different NDV strains is a rare event (Afonso, 2008) and that apparent genetic recombination in NDV may be an artifact (artificial recombination) (Song et al., 2011). Nevertheless, evidence of recombination between wild type and vaccine strains of NDV is relatively under-reported compared to other RNA viruses including avian influenza viruses (Webster et al., 1974), infectious bursal disease virus (Hon et al., 2008) and infectious bronchitis virus (Cavanagh et al., 1992).

Taken together, this study has described the application of NGS-based technology for complete genome sequencing of genotype VII NDV isolates. The protocol was able to generate consensus-level full genome sequence of five virulent NDVs obtained from outbreaks during 2004-2013 in Malaysia. The in-depth studies conducted on the different genotype VII NDV demonstrated the increase in the evolutionary distance of $\mathrm{F}$ and
HN proteins of circulating lineage 5 NDVs against lineage 2. Furthermore, this study provides the evidence of recombination between the vaccine lineage and circulating virus, which warrants the importance of continuous investigations on the genome-wide study of NDV diversity.

\section{AUTHOR CONTRIBUTIONS}

Conceived and designed the experiments: AO, DS, ST, and SY. Performed the experiments: DS and KM. Analyzed the data: DS, $\mathrm{AO}, \mathrm{ST}$, and MM. Contributed reagents/materials/analysis tools: $\mathrm{AO}$ and AI. Wrote the manuscript: DS. Critical revision: AO and MM.

\section{FUNDING}

This work was supported by an Institute of Bioscience, Higher Institution Centre of Excellence grant (IBS HICoE 6369101) from the Ministry of Education, Government of Malaysia, 
Universiti Putra Malaysia Grant No. P-IPB/2013/9425700, and Biotechnology and Biological Sciences Research Council (BBSRC) through Institute Strategic Program Grant (BB/J004448/1).

\section{REFERENCES}

Afonso, C. L. (2008). Not so fast on recombination analysis of Newcastle disease virus. J. Virol. 82, 9303-9303. doi: 10.1128/JVI.01231-08

Aldous, E. W., Mynn, J. K., Banks, J., and Alexander, D. J. (2003). A molecular epidemiological study of avian paramyxovirus type 1 (Newcastle disease virus) isolates by phylogenetic analysis of a partial nucleotide sequence of the fusion protein gene. Avian Pathol. 32, 239-257. doi: 10.1080/030794503100009783

Alexander, D. J. (1997). "Newcastle disease and avian paramyxovirus infections," in Diseases of Poultry, eds B. W. Calnek, H. J. Barnes, C. W. Beard, and L. R. McDougald (Ames, IA: Iowa State University Press), 541-569.

Ballagi-Pordány, A., Wehmann, E., Herczeg, J., Belák, S., and Lomniczi, B. (1996). Identification and grouping of Newcastle disease virus strains by restriction site analysis of a region from the F gene. Arch. Virol. 141, 243-261. doi: 10.1007/ BF01718397

Berhanu, A., Ideris, A., Omar, A. R., and Bejo, M. H. (2010). Molecular characterization of partial fusion gene and C-terminus extension length of haemagglutinin-neuraminidase gene of recently isolated Newcastle disease virus isolates in Malaysia. Virol. J. 7:183. doi: 10.1186/1743-422X-7-183

Cavanagh, D., Davis, P. J., and Cook, J. K. (1992). Infectious bronchitis virus: evidence for recombination within the Massachusetts serotype. Avian Pathol. 21, 401-408. doi: 10.1080/03079459208418858

Chambers, P., Millar, N. S., and Emmerson, P. T. (1986). Nucleotide sequence of the gene encoding the fusion glycoprotein of Newcastle disease virus. J. Gen. Virol. 67, 2685-2694. doi: 10.1099/0022-1317-67-12-2685

Cho, S. H., Kim, S. J., and Kwon, H. J. (2007). Genomic sequence of an antigenic variant Newcastle disease virus isolated in Korea. Virus Genes 35, 293-302. doi: 10.1007/s11262-007-0078-z

Chong, Y. L., Padhi, A., Hudson, P. J., and Poss, M. (2010). The effect of vaccination on the evolution and population dynamics of avian paramyxovirus-1. PLoS Pathog. 6:e1000872. doi: 10.1371/journal.ppat.1000872

Collins, M. S., Bashiruddin, J. B., and Alexander, D. J. (1993). Deduced amino acid sequences at the fusion protein cleavage site of Newcastle disease viruses showing variation in antigenicity and pathogenicity. Arch. Virol. 128, 363-370. doi: $10.1007 / \mathrm{BF} 01309446$

Commission of the European Communities (CEC) (1992). Council Directive 92/66/EC of 14 July 1992 introducing community measures for the control of Newcastle disease. Office J. Eur. Comm. L260, 1-20.

Czeglédi, A., Ujvári, D., Somogyi, E., Wehmann, E., Werner, O., and Lomniczi, B. (2006). Third genome size category of avian paramyxovirus serotype 1 (Newcastle disease virus) and evolutionary implications. Virus Res. 120, 36-48. doi: 10.1016/j.virusres.2005.11.009

de Leeuw, O., and Peeters, B. (1999). Complete nucleotide sequence of Newcastle disease virus: evidence for the existence of a new genus within the subfamily Paramyxovirinae. J. Gen. Virol. 80, 131-136. doi: 10.1099/0022-1317-80-1-131

Diel, D. G., da Silva, L. H., Liu, H., Wang, Z., Miller, P. J., and Afonso, C. L. (2012). Genetic diversity of avian paramyxovirus type 1: proposal for a unified nomenclature and classification system of Newcastle disease virus genotypes. Infect. Genet. Evol. 12, 1770-1779. doi: 10.1016/j.meegid.2012.07.012

Dortmans, J. C., Koch, G., Rottier, P. J., and Peeters, B. P. (2011). Virulence of Newcastle disease virus: what is known so far? Vet. Res. 42:122. doi: 10.1186/ 1297-9716-42-122

Dortmans, J. C. F. M., Peeters, B. P. H., and Koch, G. (2012). Newcastle disease virus outbreaks: vaccine mismatch or inadequate application? Vet. Microbiol. 160, 17-22. doi: 10.1016/j.vetmic.2012.05.003

European Union (EU) (2010). Directive 2010/63/EU of The European Parliament and of the Council of 22 September 2010 on the protection of animals used for scientific purposes. Office J. Eur. Comm. L276, 33-79. Available online at: http://eur-lex.europa.eu/LexUriServ/LexUriServ.do?uri=OJ:L:2010: 276:0033:0079:en:PDF

\section{ACKNOWLEDGMENTS}

We thank the entire technical staff of the Laboratory of Vaccine and Immunotherapeutic for their excellent assistant.

Han, G. Z., He, C. Q., Ding, N. Z., and Ma, L. Y. (2008). Identification of a natural multi-recombinant of Newcastle disease virus. Virology 371, 54-60. doi: 10. 1016/j.virol.2007.09.038

Hon, C. C., Lam, T. T., Yip, C. W., Wong, R. T., Shi, M., Jiang, J., et al. (2008). Phylogenetic evidence for homologous recombination within the family Birnaviridae. J. Gen. Virol. 89, 3156-3164. doi: 10.1099/vir.0.2008/004101-0

Khan, T. A., Rue, C. A., Rehmani, S. F., Ahmed, A., Wasilenko, J. L., Miller, P. J., et al. (2010). Phylogenetic and biological characterization of Newcastle disease virus isolates from Pakistan. J. Clin. Microbiol. 48, 1892-1894. doi: 10.1128/ JCM.00148-10

Kim, L. M., King, D. J., Curry, P. E., Suarez, D. L., Swayne, D. E., Stallknecht, D. E., et al. (2007a). Phylogenetic diversity among low-virulence Newcastle disease viruses from waterfowl and shorebirds and comparison of genotype distributions to those of poultry-origin isolates. J. Virol. 81, 12641-12653. doi: 10.1128/JVI.00843-07

Kim, L. M., King, D. J., Suarez, D. L., Wong, C. W., and Afonso, C. L. (2007b). Characterization of class I Newcastle disease virus isolates from Hong Kong live bird markets and detection using real-time reverse transcription-PCR. J. Clin. Microbiol. 45, 1310-1314. doi: 10.1128/JCM.02594-06

Leary, S., Underwood, W., Anthony, R., Cartner, S., Corey, D., Grandin, T., et al. (2013). AVMA Guidelines for the Euthanasia of Animals. Schaumburg, IL: American Veterinary Medical Association.

Locke, D. P., Sellers, H. S., Crawford, J. M., Schultz-Cherry, S., King, D. J., Meinersmann, R. J., et al. (2000). Newcastle disease virus phosphoprotein gene analysis and transcriptional editing in avian cells. Virus Res. 69, 55-68. doi: 10. 1016/S0168-1702(00)00175-1

Maminiaina, O. F., Gil, P., Briand, F. X., Albina, E., Keita, D., Andriamanivo, H. R., et al. (2010). Newcastle disease virus in madagascar: identification of an original genotype possibly deriving from a died out ancestor of genotype IV. PLoS ONE 5:e13987. doi: 10.1371/journal.pone.0013987

Martin, D. P., Williamson, C., and Posada, D. (2005). RDP2: Recombination detection and analysis from sequence alignments. Bioinformatics 21, 260-262. doi: 10.1093/bioinformatics/bth490

Miller, P. J., Decanini, E. L., and Afonso, C. L. (2010). Newcastle disease: evolution of genotypes and the related diagnostic challenges. Infect. Genet. Evol. 10, 26-35. doi: 10.1016/j.meegid.2009.09.012

Munir, M., Cortey, M., Abbas, M., Qureshi, Z. U., Afzal, F., Shabbir, M. Z., et al. (2012). Biological characterization and phylogenetic analysis of a novel genetic group of Newcastle disease virus isolated from outbreaks in commercial poultry and from backyard poultry flocks in Pakistan. Infect. Genet. Evol. 12, 1010-1019. doi: 10.1016/j.meegid.2012.02.015

Nagai, Y., Klenk, H. D., and Rott, R. (1976). Proteolytic cleavage of the viral glycoproteins and its significance for the virulence of Newcastle disease virus. Virology 72, 494-508.

Okoye, J. O., and Shoyinka, S. V. (1983). Newcastle disease in a vaccinated flock which had experienced subclinical infectious bursal disease. Trop. Anim. Health. Prod. 15, 221-225. doi: 10.1007/BF02242062

Panda, A., Huang, Z., Elankumaran, S., Rockemann, D. D., and Samal, S. K. (2004). Role of fusion protein cleavage site in the virulence of Newcastle disease virus. Microb. Pathog. 36, 1-10. doi: 10.1016/j.micpath.2003. 07.003

Perozo, F., Marcano, R., and Afonso, C. L. (2012). Biological and phylogenetic characterization of a genotype VII Newcastle disease virus from Venezuela: efficacy of field vaccination. J. Clin. Microbiol. 50, 1204-1208. doi: 10.1128/JCM. 06506-11

Qin, Z., Sun, L., Ma, B., Cui, Z., Zhu, Y., Kitamura, Y., et al. (2008). F gene recombination between genotype II and VII Newcastle disease virus. Virus Res. 131, 299-303. doi: 10.1016/j.virusres.2007.10.001

Ray, S. C. (2003). SimPlot for Windows (version 3.5.1). Baltimore, MD. Available online at: http://sray.med.som.jhmi.edu/SCRoftware/ (Accessed June 2015). 
Read, A. F., Baigent, S. J., Powers, C., Kgosana, L. B., Blackwell, L., Smith, L. P., et al. (2015). Imperfect vaccination can enhance the transmission of highly virulent pathogens. PLoS Biol. 13:e1002198. doi: 10.1371/journal.pbio.1002198

Roohani, K., Tan, S. W, Yeap, S. K., Ideris, A., Bejo, M. H., and Omar, A. R. (2015). Characterization of the genotype VII Newcastle disease virus (NDV) isolated from NDV vaccinated chickens and the efficacy of LaSota and recombinant genotype VII vaccines against challenge with velogenic NDV. J. Vet. Sci. 16, 447-457. doi: 10.4142/jvs.2015.16.4.447

Samuel, A., Nayak, B., Paldurai, A., Xiao, S., Aplogan, G. L., Awoume, K. A., et al. (2013). Phylogenetic and pathotypic characterization of Newcastle disease viruses circulating in West Africa and efficacy of a current vaccine. J. Clin. Microbiol. 51, 771-781. doi: 10.1128/JCM.02750-12

Snoeck, C. J., Ducatez, M. F, Owoade, A. A, Faleke, O. O, Alkali, B. R., Tahita, M. C., et al. (2009). Newcastle disease virus in West Africa: new virulent strains identified in non-commercial farms. Arch. Virol. 154, 47-54. doi: 10.1007/ s00705-008-0269-5

Snoeck, C. J., Owoade, A. A., Couacy-Hymann, E., Alkali, B. R., Okwen, M. P., Adeyanju, A. T., et al. (2013). High genetic diversity of Newcastle disease virus in poultry in west and central Africa: cocirculation of genotype XIV and newly defined genotypes XVII and XVIII. J. Clin. Microbiol. 51, 2250-2260. doi: 10. 1128/JCM.00684-13

Song, Q., Cao, Y., Li, Q., Gu, M., Zhong, L., Hu, S., et al. (2011). Artificial recombination may influence the evolutionary analysis of Newcastle disease virus. J. Virol. 85, 10409-10414. doi: 10.1128/JVI.00544-11

Tamura, K., Stecher, G., Peterson, D., Filipski, A., and Kumar, S. (2013). MEGA6: Molecular evolutionary genetics analysis version 6.0. Mol. Biol. Evol. 30, 2725-2729. doi: 10.1093/molbev/mst197

Tan, S. W., Ideris, A., Omar, A. R., Yusoff, K., and Hair-Bejo, M. (2010). Sequence and phylogenetic analysis of Newcastle disease virus genotypes isolated in Malaysia between 2004 and 2005. Arch. Virol. 155, 63-70. doi: 10.1007/s00705009-0540-4

Thompson, J. D., Gibson, T. J., Plewniak, F., Jeanmougin, F., and Higgins, D. G. (1997). The CLUSTAL_X windows interface: flexible strategies for multiple sequence alignment aided by quality analysis tools. Nucleic Acids Res. 25, 4876-4882.

Wang, K. C, Chen, G. Q., Jiang, W. M., Liu, S., Hou, G. Y., Yu, J. M., et al. (2013). Complete genome sequence of a hemagglutination-negative avian paramyxovirus type 4 isolated from China. Genome Announc. 1:e00045-13. doi: 10.1128/genomeA.00045-13

Webster, R. G., Isachenko, V. A., and Carter, M. (1974). A new avian influenza virus from feral birds in the USSR: recombination in nature? Bull World Health Organ. 51, 325-332.
World Animal Health Information Database Interface (WAHID) (2015). Available online at: http://www.oie.int/wahis_2/public/wahid.php/Diseaseinformation/ statusdetail (Accessed July 2015).

World Organisation for Animal Health (OIE) (2012). "Newcastle disease," in OIE Manual of Standards for Diagnostic Tests and Vaccines for Terrestrial Animals, eds S. Linnane and S. Edwards (Paris: World Organisation for Animal Health), 576-589.

Xiao, S., Nayak, B., Samuel, A., Paldurai, A., Kanabagattebasavarajappa, M., Prajitno, T. Y., et al. (2012). Generation by reverse genetics of an effective, stable, live-attenuated Newcastle disease virus vaccine based on a currently circulating, highly virulent Indonesian strain. PLOS ONE 7:e52751. doi: 10. 1371/journal.pone.0052751

Yin, Y., Cortey, M., Zhang, Y., Cui, S., Dolz, R., Wang, J., et al. (2011). Molecular characterization of Newcastle disease viruses in Ostriches (Struthio camelus L.): further evidences of recombination within avian paramyxovirus type 1. Vet. Microbiol. 149, 324-329. doi: 10.1016/j.vetmic.2010.12.004

Yu, L., Wang, Z., Jiang, Y., Chang, L., and Kwang, J. (2001). Characterization of newly emerging Newcastle disease virus isolates from the People's Republic of China and Taiwan. J. Clin. Microbiol. 39, 3512-3519. doi: 10.1128/JCM.39.10. 3512-3519.2001

Yusoff, K., and Tan, W. S. (2001). Newcastle disease virus: macromolecules and opportunities. Avian Pathol. 30, 439-455. doi: 10.1080/030794501200 78626

Zenglei, H., and Liu, X. (2015). Immune response-induced NDV pathology. Br. J. Virol. 2, 25-27. Available online at: http://smithandfranklin.com/currentissues/NDV-Induced-Immune-Pathology-in-Chickens/6/15/93/html

Zhang, R., Wang, X., Su, J., Zhao, J., and Zhang, G. (2010). Isolation and analysis of two naturally-occurring multi-recombination Newcastle disease viruses in China. Virus Res. 151, 45-53. doi: 10.1016/j.virusres.2010. 03.015

Conflict of Interest Statement: The authors declare that the research was conducted in the absence of any commercial or financial relationships that could be construed as a potential conflict of interest.

Copyright (c) 2016 Satharasinghe, Murulitharan, Tan, Yeap, Munir, Ideris and Omar. This is an open-access article distributed under the terms of the Creative Commons Attribution License (CC BY). The use, distribution or reproduction in other forums is permitted, provided the original author(s) or licensor are credited and that the original publication in this journal is cited, in accordance with accepted academic practice. No use, distribution or reproduction is permitted which does not comply with these terms. 